\title{
Applying Image Recognition Technology to Intelligent Security Management Systems
}

\author{
Y.N. Chung \\ Department of Electrical Engineering \\ National Changhua University of Education \\ Changhua, 500, Taiwan
}

T.C. Lu

Department of Electrical Engineering

National Changhua University of Education

Changhua, 500, Taiwan

\section{Y.X. Huang}

Department of Electrical Engineering, National Changhua University of Education, Changhua, 500, Taiwan

\author{
C.T. Lin \\ Department of Electrical Engineering \\ National Changhua University of Education \\ Changhua, 500, Taiwan \\ C.C. $\mathrm{Yu}$ \\ Department of Electrical Engineering \\ Da-yeh University \\ No.168, University Rd., Dacun, Changhua 51591, Taiwan
}

C.K. Li

Department of Electrical Engineering

National Changhua University of Education Changhua, 500, Taiwan

\begin{abstract}
A building safety management system based on image processing technology, which includes the license plate recognition, face recognition, and Radio Frequency Identification(RFID) systems are investigated in this paper. The system integrates three functional capabilities, which can effectively control access to user identity and to control management building security. The image technology is used to do license plate and facerecognition. In order to recognize the license plate and face thecolor space conversion, segmentation, and image processing technology is applied. Finally, the integration of RFID image processing is applied to automatic security management system. Only the identified users can pass through the gate of building to make sure the security.
\end{abstract}

Keywords-image processing technology; license plate recognition; face recognition; radio frequency identification

\section{INTRODUCTION}

The security of public space and building is paid more attention in recent years. The guard is necessary to make sure the safety of people. Most of the users still preserve the human guard. However, the human is easy to make error such as misjudgment and cost of human guard is relative highalso. Therefore, there are many public places do not have guards. In order to have safe activity space, an intelligentautomatic security management system is investigated to handle this job. Using this system, the person or the vehicle does not on the list of database is not allowed to enter the gate to enhance the people security.

In this paper, an integration system which includes Radio Frequency Identification (RFID) [1]and image processing [2-3] is applied. If people like to pass through the gate, the system will check the RFID and face recognition [4-6] by using
imagetechnology.If vehicles like to pass through the gate, the system will check the RFID, license plate [7] and face recognition by using image technology. The image processing technology which includes thecolor space conversion, segmentation, and some other image processing technology is applied in this paper.This technology is convinced to have effective control and good identity confirmation. It can integratedifferent informationeffectivelyand overcome the traditional security problems also.

An intelligent security management system is developed in this paper. The system process is shown in Figure 1.The image identification section contains the license plate recognition algorithm and face recognition algorithm. This paper presents a complex image recognition technology based on user security management system to have effective control and good identity confirmation.

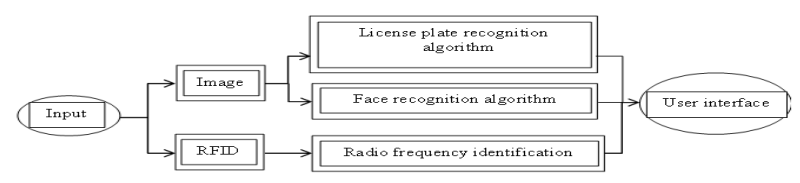

FIGURE I. SECURITYSYSTEM ARCHITECTURE

\section{LICENSE PLATE RECOGNITION ALGORITHM}

License plate recognition system uses the camera to capture images of vehicles, then transfer captured images to a computer through a series of image processing procedures. The process includes the former image processing, license plate location, license plate characters segmentation, and license plate character recognition. First, it captures a video image and does the pre-processing steps. In order to save computation time the RGB color image is converted into grayscale images. 
And then, it uses histogram equalization to enhance the image contrast and brightness adjustment, so that the image processing operations can be done at the same standard, as shown in Figure2.Because there is background noise, the system uses edge detection method and morphology to highlight the candidate block the plate and set the filter criteria to find the location of the license plate. In this paper, a vertical sober edge detection method is applied.

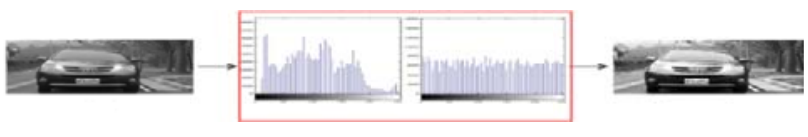

FIGURE II. IMAGE PRE-PROCESSING

Morphological processing is an important process, which is widely used in target detection, noise removal, segmentation, and block boundary capture. This paper doesn't apply additional noise elimination for simple the processing only using the morphological processing to reduce it. The closing and opening algorithm of morphological processing based on dilation and erosion are useful to fill up the vacancies of segmented foreground objects and to eliminate some noise. These two methods can let the detected objects are more intact to present.

After morphological process, images will form many different sizes of blocks, and then it will set the initial filter criteria to filter out unlikely license plate blocks. If the block area is less than 120 pixels will be removed. According to the actual experimental simulation analysis, it sets the block filter formula is shown as Eq. (1) and (2).If the image is actual location of the license plate which meets the above two conditions will be filtered out and shown in Figure 3.

Block Aspect ratio:

$$
2 \leq \text { Length / Width } \leq 2.2
$$

Block pixels:

$$
4400 \leq \text { AreaPixels } \leq 4800
$$

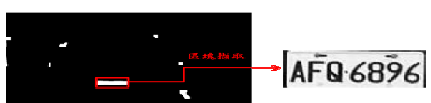

FIGURE III. LICENSE PLATE LOCATION RESULTS

After capturing the license plate position, the image noise which isless than 60 pixels will be filtered out.And then the clear license plate will be shown.Segmentation is based on projection histogram analysis to find their proper segmentation threshold values. Pixel distribution state and the cumulative amount of the image project onto the coordinate axis. Based on its distribution histogram, the algorithm identifies a local maximum or minimum of the cumulative pixel position. The algorithm setsthe segmentation threshold to achieve the purpose of segmentation. This paper uses horizontal positioning projection split upper and lower boundaries of the amendments. The vertical projection of character segmentation achieves correctposition and cut out characters, the result is shown in Figure 4.

\section{AF06896 $A 1816896$

(a)Horizontal projection(b)Vertical projection

FIGURE IV. LICENSE PLATE SEGMENTATION PROJECTIONS

After cutting through the vertical projection, it gives the license plate characters in each block of the image. This algorithm splits character image block after normalization to image size, the results are shown in Figure 5. And then the algorithm starts the character recognition process.In this paper, a model comparison method is applied to complete the final alignment results. When the image size is specified, it will calculate the correlation coefficient shown as Eq. (3).Where $\mathrm{R}$ is the correlation coefficient, $A_{m n}$ is standard model grayscale, $\bar{A}$ is the average value of standard model grayscale, $B_{m n}$ is the test image gray order, and $\bar{B}$ is the average value of test image grayscale. The highest correlation coefficient of the object is the comparison results.

\section{A8 8686}

FIGURE V. CHARACTER NORMALIZATION RESULTS

$$
R=\frac{\sum_{m} \sum_{n}\left(A_{m n}-\bar{A}\right)\left(B_{m n}-\bar{B}\right)}{\sqrt{\left(\sum_{m} \sum_{n}\left(A_{m n}-\bar{A}\right)^{2}\right)\left(\sum_{m} \sum_{n}\left(B_{m n}-\bar{B}\right)^{2}\right)}}
$$

\section{FACE RECOGNITION ALGORITHM}

Face recognition algorithm consists of three parts, which are human face location detection, facial image capture and formalize the region, and principal component analysis comparison. After the face images are captured, the system uses color space conversion and analyzes the region of face. The Otsu's methodis used to make image segmentation to have more precise position of human face. The face recognition is based on the identification of the principal component analysis framework to establish facial feature space. Finally, the algorithm uses Euclidean distance to determine the size of the human face identity.

Face location detection step consists of two parts, which are human face image pre-processing and the human face correction and calibration. First, the pretreatment step is the color space conversion and the image analysis to aim the face image area.It will detect the human face image region. Subsequently, the system uses morphological processing to remove unwanted component and then more accurate face position can be marked.

Traditional RGB color images are susceptible if light intensity is changed. In this paper, the $L^{*} a^{*} b^{*}$ color space conversion is chosen todo human face detection component analysis. The $\mathrm{L}^{*} \mathrm{a}^{*} \mathrm{~b}^{*}$ color space for the color opponent space is based on CIE-XYZ color space coordinates. $\mathrm{L}^{*}$ represents the luminance component, $\mathrm{a}^{*}$ represents the red/green color component, and $b^{*}$ represents the yellow/blue component. RGB color images cannot be directly converted into $\mathrm{L}^{*} \mathrm{a}^{*} \mathrm{~b}^{*}$ color images.It uses thetransformation 
matrixshown as Eq. (4)to convert it to CIE-XYZ color space, and then find its $\mathrm{X}, \mathrm{Y}, \mathrm{Z}$ values.The CIE- LAB color space can be obtained by using Eq. (5) to Eq. (8).

$$
\begin{gathered}
{\left[\begin{array}{c}
X \\
Y \\
Z
\end{array}\right]=\left[\begin{array}{lll}
0.412453 & 0.357580 & 0.180423 \\
0.212671 & 0.715160 & 0.072169 \\
0.019334 & 0.119193 & 0.950227
\end{array}\right.} \\
L^{*}=\left\{\begin{array}{r}
116 \times\left(\frac{Y}{Y_{n}}\right)^{\frac{1}{3}}-16, \quad \frac{Y}{Y_{n}}>0.008856 \\
903.3 \times \frac{Y}{Y_{n}}, \quad \text { otherwise }
\end{array}\right. \\
a^{*}=500 \times\left(f\left(\frac{X}{X_{n}}\right)-f\left(\frac{Y}{Y_{n}}\right)\right) \\
b^{*}=200 \times\left(f\left(\frac{Y}{Y_{n}}\right)-f\left(\frac{Z}{Z_{n}}\right)\right)
\end{gathered}
$$

Where $\mathrm{X}_{\mathrm{n}}=0.9515, \mathrm{Y}_{\mathrm{n}}=1.0000, \mathrm{Z}_{\mathrm{n}}=1.0886$, and $f(t)$ is shown as Eq. (8).

$$
f(t)=\left\{\begin{array}{cc}
t^{\frac{1}{3}}, t>0.008856 \\
7.787 \times t+\frac{16}{116} \quad, \quad \text { otherwise }
\end{array}\right.
$$

The conversion result is shown in Figure 6. Figure 7is the $L^{*} a^{*} b *$ color space components of human face image.

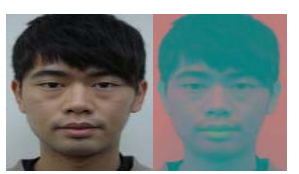

(a)RGB image(b) $\mathrm{L} * \mathrm{a} * \mathrm{~b} *$ image

FIGURE VI. RGB IMAGE AND L*A* B* IMAGE

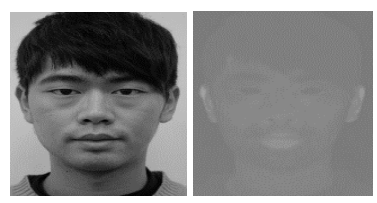

(a)Luminance component(b)R/G component

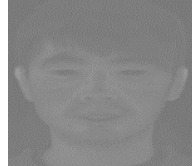

(c)Y/B component

FIGURE VII. L*A* B* COLOR SPACE COMPONENTS

In this paper, the Otsu's image area segmentation method is used.By Otsu's image region segmentation method can automatically analyze the optimal segmentation threshold of human face images.The face segmentation histogram analysis results is shown in figure 8 , which are the components of the color space by a* and $b^{*}$ conversion. The red dotted line is the threshold values for the analyzed position. Based on the image results, the Otsu's image region segmentation method can effectively separate the face component.

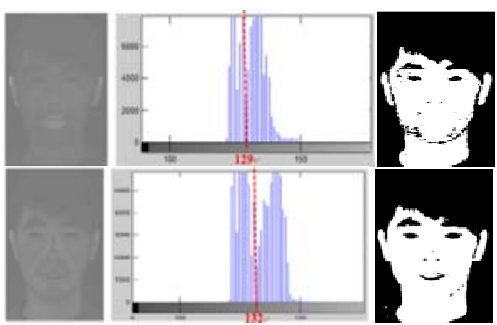

FIGURE VIII.

FACE SEGMENTATION HISTOGRAM ANALYSIS RESULTS

Let these two image region segmentations be intersected and the two images will extract a common component. It will obtain a more accurate face region of the image component shown in Figure 9(a). Subsequently, the morphological process is applied to treat the face image. The result of image after morphological processing is shown in Figure 9(b). Figure 9(c) is the corresponding RGB color image position in which the background image has been removed. Afterthe corresponding position of the face image is obtained, it only remain the image characteristic such as eyebrows, eyes, nose, and lips, which are different based on different face. After positioning the image block, which can identify a face in the image composition block size and the range of coordinates. The face component block range coordinates (X1, Y1) and (X2, Y2), can be scaled by using Eq. (9)to Eq. (12) to obtain correction range coordinate area (Left, Top) and (Right, Bottom). Its relative position is shown in the Figure 10(a), and the actual marked face is shown in Figure 10(b).The human face is not fixed, so the face block size image should be normalized to same size.

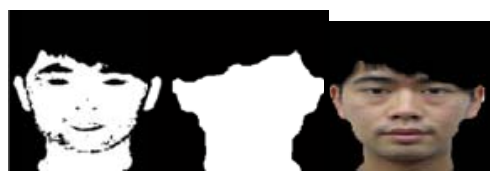

(a)Intersectionimage(b)Image after morphological (c)RGBimage position

FIGURE IX. FACE SEGMENTATION HISTOGRAM ANALYSIS RESULTS

$$
\begin{aligned}
& \text { Top }=Y_{1}+\text { Length } / 0.05(9) \text { Bottom }=Y_{1}+\text { Length } / 1.3 \\
& \text { Left }=X_{1}+\text { Width } / 8(11) \text { Right }=X_{2}-\text { Width } / 8 \\
& \text { (a) }
\end{aligned}
$$

(a)Correction region of face (b) Mark of human face FIGURE X. CORRECTION DIAGRAM OF THE HUMAN FACE 
After normalizing the human face image size, this paper analyzesthrough the main ingredient to complete face recognition algorithm. It is widely used in two-dimensional face recognition. After conversion you can use less data to replace the original data, so the advantage of its method can reduce the dimension of image data and can quickly analyze the results. Finally, the algorithm uses the Euclideandistance to find the closest results fromtraining sample library. The difference between theimagesof human face is projected onto the feature spaceand to find the weight vector in space. Based on the computation results ofEuclidean distance shown as Eq. (13), the systemwill find the same person's face image between the two vectors have the smallest value.

$$
D(x, y)=\sqrt{\left(x_{1}-y_{1}\right)^{2}+\left(x_{2}-y_{2}\right)^{2}+\ldots+\left(x_{n}-y_{n}\right)^{2}}
$$

\section{RFID AND USER INTERFACE}

In thissystem onepassive $13.56 \mathrm{MHz}$ frequency specification of RFID is chosen. The system can directly integrate with the Lab View user interface. First, it needs to set port and transport protocol to establish the reader and the computer transmission channel. After communication interface setup is completed it can build RFID encoding list in the system and it will check the user code to confirm whether he is on list or not. The system also displays the user information in computer. Then theuser's identity can be checked easily. Finally, this study integrates the three functions which are license plate recognition, face recognition, and RFID to identify the users. Theidentification resultswill transmit to the user interface in Lab VIEW and it will display related information. The security center can monitor the results of operation for each identification sample. Based on real time experiment results, the successful rate is over $90 \%$.

\section{CONCLUSION}

This study presents an intelligent security management systemwhich integrateslicense plate recognition algorithm, face recognition algorithm, and radio frequency identification technology to make the security monitor automatically. The system constructs anintelligent image processing technology which can identify the license plate and human face. This system has automatic identification and specific user identity functions. Moreover, the system willtransmit identification results to the management interface usingLab VIEW and display related information. The proposed system is convinced that it is not expensive but it has efficient security management capability.

\section{ACKNOWLEDGEMENT}

This work was supported by the National Science Council under Grant MOST 103-2221-E-018-017.

\section{REFERENCES}

[1] J M. Deriche, M. Mohandes , "A Hybrid RFID-LPR System for Vehicle Access Control during Pilgrimage Season in Saudi Arabia,” Systems, Signals and Devices (SSD), 2012 9th International Multi-Conference on, pp.1-6, 2012.

[2] Bin Zhang, Kuizhi Mei, and Nanning Zheng, "Reconfigurable Processor for Binary Image Processing," Circuits and Systems for Video Technology, IEEE Transactions on, Volume 23 , Issue 5 , pp.823-831,
2013.

[3] Sang-Heon Lee, Dong-Ju Kim and Jin-Ho Cho, "Illumination-Robust Face Recognition System,” Transactions on IEEE Consumer Electronics, Vol. 58, Issue. 3, pp.963-970, August 2012.

[4] Shih-Ming Huang and Jar-Ferr Yang, "Improved Principal Component Regression for Face Recognition Under Illumination Variations," Transactions on IEEE Signal Processing Letters, Vol. 21, Issue. 12, pp.4830-4843, Dec 2012.

[5] G. PrabhuTeja and S. Ravi, "Face Recognition using Subspaces Techniques,” Recent Trends In Information Technology (ICRTIT), 2012 International Conference on, pp.103-107, 2012.

[6] N.K.Patil, S. Vasudha, and L.R. Boregowda, “A Novel Method for Illumination Normalization for Performance Improvement of Face Recognition System,” Electronic System Design (ISED), 2013 International Symposium on, pp.148-152, 2013.

[7] Judith Sen E,Deepa Merlin Dixon K,Ansy and Jinesh K J, “Advanced License Plate Recognition System For Car Parking,” Embedded Systems (ICES), 2014 International Conference on, pp.162-165, 2014. 